\title{
Urachal sinus
}

INSERM

\section{Source}

INSERM. (1999). Orphanet: an online rare disease and orphan drug data base. Urachal SinUS. ORPHA:431344

Urachal sinus is a type of congenital urachal anomaly (see this term) resulting from the failure of the umbilical end of the urachus to close, without continuity to the bladder, and that is usually asymptomatic but can present with continuous cloudy umbilical discharge, tender midline infraumbilical mass and fever when infected. 